\title{
Produção de sementes de espécies condimentares: situação atual e perspectivas
}

\author{
Seed production of spice species: current situation and perspectives \\ Producción de semillas de especies de especias: situación actual y perspectivas
}

Recebido: 27/09/2021 | Revisado: 03/10/2021 | Aceito: 04/10/2021 | Publicado: 05/10/2021

\author{
Henrique Berle \\ ORCID: https://orcid.org/0000-0002-4094-4642 \\ Universidade Federal de Pelotas, Brasil \\ E-mail: Henriqueberle@ hotmail.com \\ Francine Bonemann Madruga \\ ORCID: https://orcid.org/0000-0001-7202-7462 \\ Universidade Federal de Pelotas, Brasil \\ E-mail: francinebonemann@hotmail.com \\ Júlio Francisco Uriarte \\ ORCID: https://orcid.org/0000-0002-6603-8007 \\ Universidade Federal de Pelotas, Brasil \\ E-mail: jfuriarte@gmail.com \\ Edinaldo Cesar Tormes \\ ORCID: https://orcid.org/0000-0001-7390-3794 \\ Universidade Federal de Pelotas, Brasil \\ E-mail: edinaldo.tormes@outlook.com \\ Carem Rosane Coutinho Saraiva \\ ORCID: https://orcid.org/0000-0002-0810-2402 \\ Universidade Federal de Pelotas, Brasil \\ E-mail: caremsaraiva@ hotmail.com \\ Benhur Schwartz Barbosa \\ ORCID: https://orcid.org/0000-0002-8265-4598 \\ Universidade Federal de Pelotas, Brasil \\ E-mail: benhursb97@outlook.com \\ Mateus da Silveira Pasa \\ ORCID: https://orcid.org/0000-0003-0481-0206 \\ Universidade Federal de Pelotas, Brasil \\ E-mail: mateus.pasa@gmail.com \\ Lilian Vanussa Madruga de Tunes \\ ORCID: https://orcid.org/0000-0001-7562-1926 \\ Universidade Federal de Pelotas, Brasil \\ E-mail: lilianmtunes@yahoo.com.br \\ Andréia da Silva Almeida \\ ORCID: https://orcid.org/0000-0003-3169-6787 \\ Universidade Federal de Pelotas, Brasil \\ E-mail: andreiasalmeida@yahoo.com.br
}

\begin{abstract}
Resumo
Os condimentos possuem uma importância mundial devido a suas mais diversas formas de usabilidade, seja como alimentos, aromáticas ou medicinais. Ademais, movimentam quantias relevantes no mercado econômico e a produção de sementes condimentares é uma atividade que tem capacidade de impulsionar o desenvolvimento de uma região inteira, e ainda possui um forte apelo econômico e social, gerando emprego e renda na agricultura graças à elevada demanda de mão-de-obra. A presente revisão tratará sobre a produção de sementes das seguintes plantas condimentares: cebola, coentro, salsa, pimenta-do-reino e têm como objetivo descrever o cenário, produção e perspectivas futuras da produção das mesmas. Consoante a situação atual das espécies citadas acima, observa-se que, embora o grande leque de espécies condimentares, essas são pouco exploradas no Brasil, fator esse que é redigido pela lei da oferta e demanda. Mas as perspectivas são que a produção das mesmas venha a ter cada vez mais representatividade no mercado, em razão da crescente procura por essas espécies.
\end{abstract}

Palavras-chave: Condimentos; Produção de sementes; Alternativa; Importação.

\section{Abstract}

Spices are of global importance due to their most diverse forms of usability, whether as food, aromatic or medicinal. Furthermore, they move relevant amounts in the economic market and the production of spice seeds is an activity that has the capacity to boost the development of an entire region, and still has a strong economic and social appeal, generating employment and income in agriculture thanks to the high demand for labor. This review will deal with the seed production of the following spice plants: onion, coriander, parsley, black pepper and aim to define the scenario, 
production and future prospects for the production of the evaluations. Depending on the current situation of the species mentioned above, it is observed that, despite the wide range of spice species, these are little explored in Brazil, a factor that is written by the law of supply and demand. But the perspectives are that their production is becoming more and more representative in the market, due to the growing demand for these species.

Keywords: Condiments; Seed production; Alternative; Import.

\section{Resumen}

Las especias son de importancia mundial debido a sus más diversas formas de usabilidad, ya sea como alimento, aromático o medicinal. Ademais, movimentam quantias relevante no mercado econômico e a produção de sementes condimentares é uma atividade que tem capacidade de impulsionar o desenvolvimento de uma região inteira, e ainda possui um forte apelo econômico e social, gerando emprego e renda na agricultura graças à elevada demanda de mano de obra. Esta revisión abordará la producción de semillas de las siguientes plantas de especias: cebolla, cilantro, perejil, pimienta negra y tendrá como objetivo definir el escenario, la producción y las perspectivas futuras para la producción de las evaluaciones. Dependiendo de la situación actual de las especies mencionadas anteriormente, se observa que, a pesar de la amplia gama de especies de especias, estas son poco exploradas en Brasil, factor que está escrito por la ley de oferta y demanda. Pero las perspectivas son que su producción sea cada vez más representativa en el mercado, debido a la creciente demanda de estas especies.

Palabras clave: Condimentos; Producción de semillas; Alternativa; Importación.

\section{Introdução}

Existem vários relatos na história do uso de plantas condimentares de diversas formas, como por exemplo: afrodisíaca, na conservação de alimentos, medicinais, rituais místicos, entre outros. Atualmente, essas espécies são de suma importância na alimentação e na economia de diversos povos ao redor do mundo (Shultes \& Hofman, 2000; Nepomuceno, 2005; Laws, 2013; Savage, 2017). As plantas condimentares, também chamadas de "especiarias" ou "temperos", tiveram grande influência na história mundial, sendo alvo da atenção de muitos povos, com importante papel inclusive nos grandes descobrimentos dos séculos XV e XVI (Embrapa Agroindústria Tropical, 2013).

Segundo a Organização da Nações Unidas para a Alimentação e Agricultura (FAO, 1994), especiarias podem ser produtos vegetais como folhas, flores, sementes e raízes que são ricas em óleos essenciais e princípios aromáticos. Além disso, são usados principalmente como condimentos.

Muitas das especiarias utilizadas pelos povos da antiguidade (pimenta-do-reino, cravo, canela, noz-moscada, ervadoce e o açafrão-da-terra) ainda fazem parte dos condimentos de maior representatividade no cenário mundial (FAO, 2005). Mesmo no Brasil, detentor da maior biodiversidade do mundo, estes condimentos estão entre os mais frequentes na preparação dos alimentos (Tomchinsky, 2017).

Existe uma diferença entre erva e especiaria. As ervas são plantas apreciadas por suas propriedades medicinais, palatáveis e aromáticas, enquanto as especiarias são um subgrupo das ervas. Não há um consenso botânico sobre a diferença de erva e especiaria, mas, na gastronomia, podem ser distinguidas como ervas, as plantas que são utilizadas as partes como as folhas, os caules ou as flores e especiarias as plantas em que são utilizadas as sementes, as raízes, os frutos, bagas e cascas (Embrapa Agroindústria Tropical, 2013) e ainda existem as plantas que podem ser caraterizadas tanto como erva, como especiaria, por exemplo, o coentro, que a folha é uma erva, enquanto a semente é uma especiaria. Ainda segundo (Embrapa Agroindústria Tropical, 2013), as ervas são plantas de regiões temperadas, enquanto as especiarias, em sua maioria, são provenientes dos trópicos. Adiciona-se ainda que as ervas geralmente são verdes e de sabor sutil; a coloração das especiarias varia do preto a tons de marrom e vermelho, com sabor marcante e pungente.

O mercado de plantas medicinais, aromáticas e condimentares é crescente, porém sofre diversas variações relacionadas a lei da oferta e demanda. O Brasil por sua vez importa muito mais do que exporta e isso acaba afetando diretamente nos preços desses produtos e ainda tem o efeito da preferência pelos consumidores, que exigem cada vez mais alimentos diferenciados tanto em tamanho, qualidade, coloração, forma, aroma e aspectos nutricionais (Purquerio, 2011; Veltri, 2017). 


\section{Metodologia}

Conforme (Pereira et al., 2018) ao elaborar um artigo científico está se relatando resultados de alguma descoberta, experiência realizada, descrevendo algum caso, descrevendo algum fenômeno ocorrido ou ainda fazendo uma revisão bibliográfica em relação a algum tema. Nesse sentido, o método empregado para a realização da presente pesquisa foi a revisão bibliográfica.

\subsection{Situação atual da produção de sementes de espécies condimentares}

A Organização das Nações Unidas para Alimentação e Agricultura (FAO), dispõem informações sobre a produção agrícola de vários países, e nessa lista estão incluídas culturas mais expressivas na questão da área produzida e quantidade. O Brasil aparece apenas como grande produtor de hortaliças como alho, cebola e pimenta (FAOSTAT, 2021).

Os principais estados produtores de sementes de espécies condimentares estão localizados na região Sudeste e Sul do país. A localização está diretamente relacionada a condições na produção de sementes e ao escoamento da produção (Tomchinsky, 2017). Na tabela abaixo encontram-se algumas plantas condimentares que possuem sementes e mudas e que são encontradas em catálogos de empresas na internet (Tabela 1).

Tabela 1: Espécies de plantas condimentares encontradas em catálogos.

\begin{tabular}{llc}
\hline Nome Comum & Nome científico & Sementes p/grama \\
\hline Coentro & Coriandrum sativum & 130 \\
Cebolinha & Allium schoenoprasum & 500 \\
Pimenta & Capsicum spp & 200 \\
Cebola & Allium cepa & 260 \\
Salsa & Petroselinum sp & 400 \\
Alecrim & Rosmarinus officinalis L & 1000 \\
Anis ou Erva Doce & Pimpinella anisum L & 200 \\
Cebolinha Verde Fina & Allium fistulosum L & 700 \\
Cerefólio & Anthriscus cerefolium (L.) Hoffm. & 450 \\
Cominho & Carum carvi L & 425 \\
Endro ou Aneto & Anethum graveolens L. & 900 \\
Funcho ou Erva Doce & Foeniculum vulgare Mill & 240 \\
Manjericão ou Alfavaca & Ocimum basilicum L. & 650 \\
Manjerona & Origanum majorana L. 4 & 4500 \\
Orégano & Origanum vulgare L. & 7500 \\
Sálvia para Tempero & Salvia officinalis L & 120 \\
Segurelha & Satureja hortensis L. & 1800 \\
Tomilho ou Timo & Thymus vulgaris L & 6000 \\
\hline
\end{tabular}

Fonte: www.sementesfeltrin.com.br.

A partir da pesquisa desenvolvida, foram encontrados poucos dados referentes a produção de sementes desses condimentos, porém no site do Ministério da Agricultura, Pecuária e Abastecimento (SIGEF, 2021), referente a indicadores, são encontrados alguns dados referentes a produção de sementes das espécies condimentares, como o número de campos inscritos, área total (ha) e produção estimada (Tabela 2). 
Tabela 2: Total de campos inscritos de algumas espécies condimentares.

\begin{tabular}{llll}
\hline Nome científico & Total de Campos 2020/2021 & Área (ha) & Produção estimada (ton) \\
\hline Allium cepa L. & 33.142 & $2.825 .786,91$ & $12.569 .444,84$ \\
Allium fistulosum L. & 1 & 0,3 & 0,03 \\
Anethum graveolens L. & 1 & 0,5 & 0,05 \\
Anthriscus cerefolium (L.) Hoffm & 1 & 0,2 & 0,02 \\
Capsicum annuum L. & 69 & 5,09 & 0,78 \\
Carum carvi L. & 1 & 0,2 & 0,03 \\
Coriandrum sativum & 4 & 1,6 & 2,6 \\
Cuminum cyminum L. & 1 & 0,1 & 0,02 \\
Origanum vulgare L. & 3 & 0,21 & 0,05 \\
Pimpinella anisum L. & 1 & 0,3 & 0,03 \\
Salvia officinalis L. & 1 & 0,1 & 0,05 \\
Satureja hortensis L. & 2 & 0,11 & 0,02 \\
Thymus vulgaris L. & 2 & 0,23 & 0,03 \\
\hline
\end{tabular}

Fonte: SIGEF (2021).

A maioria das espécies apresentadas na (Tabela 1) estão presentes na (Tabela 2) e possuem campos de produção inscritos e homologados no sistema legal de produção de sementes do Brasil. No entanto, as plantas condimentares de alho, cebola e pimenta, foram as únicas em que foram encontrados dados referentes a produção final, ou seja, a produção que é feita pelo produtor de sementes e é comercializada para os produtores ou agricultores para posterior cultivo e venda. A maioria das espécies condimentares se propaga por sementes e podem ser encontradas em revendedores de sementes olerícolas ou em lojas especializadas, e geralmente, nas embalagens das sementes, são encontradas instruções sobre época de plantio, solo e adubação.

Para a presente revisão, considerando a importância dos condimentos, serão abordadas apenas quatro espécies de plantas condimentares, algumas possuem representatividade na quantidade produzida como por exemplo a cebola e outras que possuem um valor não relacionado a quantidade produzida, mas sim a importância na gastronomia e benefícios que podem trazer a saúde humana, como por exemplo a salsa, coentro e pimenta-do-reino.

\section{Resultados e Discussão}

\subsection{Salsa}

A salsa (Petroselinum crispum), pertence à família Apiaceae, é uma hortaliça condimentar de origem mediterrânea, sendo está uma planta herbácea. Além de ter uma grande importância econômica devido a seus fins medicinais, a salsa é uma das plantas condimentares mais populares da gastronomia mundial, sendo uma excelente fonte de vitamina A e C, niacina, riboflavina, cálcio, ferro e fósforo (Factor et al., 2008). Ademais, a salsa é uma hortaliça que não atinge sua importância por seu volume ou valor de comercialização, mas pela ampla utilização comercial como condimento (Rodrigues et al., 2008).

No Registro Nacional de Cultivares (RNC), existe registro de 47 cultivares de salsa, sendo 7 novas cultivares dos anos 2017 a 2021, o que demonstra que existem cultivares novas sendo pesquisadas e que as empresas estão em busca de lançarem materiais genéticos cada vez mais adaptadas a características desejáveis pelos agricultores.

No Brasil, a produção de sementes de salsa na safra 2020/2021 têm como o Rio Grande do Sul o único estado produtor de sementes e os municípios produtores são Candiota, como o município mais representativo, seguido de Canguçu, Hulha Negra e Pinheiro Machado. Na safra 2020/2021 ao total foram 24 campos inscritos com uma área de 86,31 hectares e 
uma produção estimada em 65,97 toneladas (SIGEF, 2021).

A tendência é que o mercado de plantas condimentares, que inclui a salsa, aumente cada vez mais, pois são alimentos importantes para composição de dietas saudáveis para o ser humano, com riqueza de micronutrientes, fibras e outros componentes importantes para o organismo (Gonçalves, 2016). Além de serem muito importantes para a agricultura familiar em nosso país.

\subsection{Coentro}

O coentro (Coriandrum sativum L.), pertencente à família Apiaceae, é uma hortaliça-condimento de ciclo anual amplamente consumida no Brasil e de acordo com (Oliveira et al., 2005) o coentro é cultivado por pequenos produtores, em hortas domésticas, escolares e comunitárias. Também é comercializada em feiras livres e supermercados, mas também há produção de frutos, utilizados nas indústrias alimentícia e cosmética. Todas as partes da planta são comestíveis, mas também é comum o seu uso culinário condimentar in natura, quando a cultura é voltada para a obtenção de massa verde. Além de condimento, os frutos secos são usados na indústria farmacêutica e cosmética (Daflon et al., 2014).

No RNC temos disponíveis 58 cultivares de coentro, sendo 12 cultivares recentes do ano de 2015 a 2019 (MAPA, 2021). Na safra 2020, segundo dados do MAPA (SIGEF, 2021), os estados que mais possuem campos de produção de sementes são RS, BA, PE. Mas também há produção de sementes nos estados de SP, GO e MG. O RS é líder desse ranking com 52 campos de produção de sementes, seguido da Bahia com 48 e Pernambuco com 8 campos.

Segundo (Sousa, et al., 2011) devido a poucas cultivares estarem disponíveis no mercado e por falta de um programa regional para avaliação de genótipos que visasse identificar e recomendar as variedades que mais se adaptam as diversas condições ambientais, muito dos materiais são de procedência desconhecida, sendo as sementes produzidas pelos próprios agricultores, com um baixo nível tecnológico. Em geral, essas sementes apresentam baixa qualidade fisiológica (Oliveira et al., 2007).

No Brasil, há grande carência de dados estatísticos sobre a produção final de coentro, ou seja, a produção que é comercializada ou produtor final e isso se explica, em parte, pelo fato de ser produzido em pequenas propriedades (EMBRAPA, 2016).

\subsection{Cebola}

A cebola (Allium cepa L.), pertencente à família Alliaceae é uma olerícola difundida em grande parte do globo, originária do Afeganistão, Irã e partes do Sul da antiga União Soviética, esta cultura é principalmente destinada à produção de bulbos e sementes (Goldman et al., 2000; Fritsch e Friesen, 2002). Desde a antiguidade era usada em razão de suas propriedades terapêuticas e medicinais. Apresenta atividade antioxidante, anti-inflamatória, de proteção cardíaca, analgésica, antialérgica, antidiabética, etc. Muitas dessas propriedades medicinais vêm do seu óleo essencial à base de enxofre, que dá origem ao sabor e cheiro característicos da cebola. Na culinária é utilizada como tempero e condimento (EMBRAPA, 2013).

A produção de Allium cepa L. é amplamente distribuída no mundo, sendo a China campeã desse ranking, seguidos da Índia, Estados Unidos e Egito. Os principais países produtores de cebola e suas respectivas participações na produção estão apresentados na (Tabela 3). 
Tabela 3: Ranking dos maiores produtores de cebola no mundo e sua produção em toneladas.

\begin{tabular}{cccccccccc}
\hline Ano/País & China & Índia & EUA & Egito & Turquia & Paquistão & Sudão & Total \\
\hline 2013 & 22.300 .000 & 19.299 .000 & 3.159 .350 & 1.093 .230 & 1.904 .846 & 1.660 .740 & 1.037 .000 & 50.454 .166 \\
2014 & 22.529 .452 & 19.401 .680 & 3.166 .740 & 2.505 .189 & 1.790 .000 & 1.740 .184 & 1.425 .060 & 52.558 .305 \\
2015 & 23.384 .254 & 18.927 .000 & 3.413 .100 & 3.049 .607 & 1.879 .189 & 1.671 .012 & 1.583 .400 & 53.907 .562 \\
2016 & 23.758 .914 & 20.931 .000 & 3.800 .670 & 2.458 .618 & 2.120 .581 & 1.736 .361 & 1.583 .900 & 56.390 .044 \\
2017 & 24.142 .073 & 22.427 .000 & 3.737 .100 & 2.965 .218 & 2.175 .911 & 1.833 .160 & 1.599 .736 & 58.880 .198 \\
2018 & 24.525 .232 & 23.262 .000 & 3.284 .420 & 3.067 .214 & 1.930 .695 & 2.119 .675 & 1.717 .273 & 59.906 .509 \\
2019 & 24.908 .392 & 22.819 .000 & 3.170 .270 & 3.081 .047 & 2.200 .000 & 2.079 .593 & 1.919 .308 & 60.177 .610 \\
\hline Variação (\%) & 12 & 18 & 0 & 182 & 15 & 25 & 85 \\
\hline
\end{tabular}

Fonte: FAOSTAT (2021).

O Brasil aparece somente na $14^{\circ}$ posição e possui uma representatividade no valor das exportações de $0,05 \%$ e importações de 1,50\% (FAOSTAT, 2021). No período de 2013 a 2019, a produção ou área plantada diminuiu (-15,5\%) e a área colhida $(-16,1 \%)$ (Tabelas 4 e 5$)$.

Tabela 4: Área plantada ou destinada a colheita de cebola (hectares).

\begin{tabular}{ccccccccc}
\hline UF/ANO & 2013 & 2014 & 2015 & 2016 & 2017 & 2018 & 2019 & Variação (\%) \\
\hline SC & 19.029 & 19.351 & 21.298 & 21.523 & 19.671 & 17.039 & 17.237 & $-9,4$ \\
RS & 9.963 & 9.755 & 8.714 & 8.010 & 7.899 & 6.809 & 6.710 & $-32,7$ \\
BA & 4.958 & 9.273 & 9.295 & 9.517 & 5.730 & 5.494 & 6.181 & 24,7 \\
SP & 5.506 & 5.646 & 5.148 & 5.651 & 5.145 & 4.567 & 4.469 & $-18,8$ \\
PR & 7.093 & 5.894 & 5.259 & 5.261 & 5.617 & 5.072 & 4.259 & $-40,0$ \\
MG & 3.145 & 3.109 & 3.323 & 3.321 & 3.564 & 3.351 & 3.308 & 5,2 \\
GO & 1.812 & 2.400 & 2.335 & 2.549 & 2.250 & 2.291 & 2.603 & 43,7 \\
PE & 4.507 & 2.725 & 1.370 & 1.120 & 817 & 1.851 & 1.822 & $-59,6$ \\
RN & 820 & 720 & 510 & 470 & 215 & 500 & 1.200 & 46,3 \\
ES & 303 & 606 & 346 & 406 & 308 & 322 & 327 & 7,9 \\
DF & 248 & 155 & 141 & 275 & 520 & 300 & 300 & 21,0 \\
PB & 115 & 141 & 147 & 76 & 60 & 156 & 191 & 66,1 \\
CE & 80 & 44 & 30 & 28 & - & - & 52 & $-35,0$ \\
PA & - & - & - & 15 & 20 & 20 & 20 & 33,3 \\
PI & 8 & 11 & 7 & 6 & 6 & 7 & 4 & $-50,0$ \\
Total & 57.587 & 59.830 & 57.923 & 58.228 & 51.822 & 47.779 & 48.683 & $-15,5$ \\
\hline
\end{tabular}

Fonte: SIDRA (2021). 
Tabela 5: Área colhida de cebola (hectares).

\begin{tabular}{clllllllc}
\hline UF/ANO & 2013 & 2014 & 2015 & 2016 & 2017 & 2018 & 2019 & Variação (\%) \\
\hline SC & 19.029 & 19.311 & 20.066 & 21.423 & 19.596 & 17.039 & 17.177 & $-9,7$ \\
RS & 9.963 & 9.755 & 8.714 & 7.560 & 7.899 & 6.809 & 6.710 & $-32,7$ \\
BA & 4.958 & 9.273 & 9.295 & 9.517 & 5.662 & 5.494 & 5.931 & 19,6 \\
SP & 5.371 & 5.511 & 5.148 & 5.651 & 5.145 & 4.567 & 4.469 & $-16,8$ \\
PR & 7.093 & 5.894 & 5.245 & 5.127 & 5.617 & 5.067 & 4.132 & $-41,7$ \\
MG & 3.145 & 3.109 & 3.323 & 3.321 & 3.564 & 3.351 & 3.308 & 5,2 \\
GO & 1.812 & 2.400 & 2.335 & 2.549 & 2.250 & 2.291 & 2.535 & 39,9 \\
PE & 4.457 & 2.260 & 1.370 & 995 & 812 & 1.721 & 1.790 & $-59,8$ \\
RN & 820 & 720 & 510 & 470 & 215 & 500 & 1.200 & 46,3 \\
ES & 303 & 606 & 346 & 406 & 308 & 322 & 327 & 7,9 \\
DF & 248 & 155 & 141 & 205 & 520 & 300 & 300 & 21,0 \\
PB & 115 & 141 & 147 & 76 & 60 & 156 & 191 & 66,1 \\
CE & 80 & 44 & 30 & 28 & - & - & 52 & $-35,0$ \\
PA & - & - & - & 15 & 20 & 20 & 20 & 33,3 \\
PI & 8 & 11 & 7 & 6 & 6 & 7 & 4 & $-50,0$ \\
\hline Total & 57.402 & 59.190 & 56.677 & 57.349 & 51.674 & 47.644 & 48.146 & $-16,1$ \\
\hline
\end{tabular}

Fonte: SIDRA (2021).

No entanto a produção de cebola em toneladas teve uma estabilidade $(1,2 \%)$ e o rendimento médio da produção variou $(-7,7 \%) \mathrm{kg} /$ hectare (Tabelas 6 e 7$)$. 
Tabela 6: Produção de cebola em toneladas.

\begin{tabular}{ccccccccc}
\hline UF/ANO & 2013 & 2014 & 2015 & 2016 & 2017 & 2018 & 2019 & Variação (\%) \\
\hline SC & 496.973 & 474.709 & 339.451 & 546.259 & 431.759 & 470.873 & 457.221 & $-8,0$ \\
BA & 112.858 & 323.120 & 282.904 & 291.175 & 265.465 & 242.789 & 242.807 & 115,1 \\
MG & 172.461 & 171.485 & 195.528 & 185.524 & 200.552 & 189.282 & 192.443 & 11,6 \\
SP & 190.268 & 203.493 & 197.070 & 222.041 & 218.012 & 168.668 & 171.309 & $-10,0$ \\
GO & 120.600 & 85.280 & 105.225 & 117.410 & 130.400 & 121.170 & 169.048 & 40,2 \\
RS & 159.735 & 171.179 & 147.439 & 144.357 & 175.716 & 138.435 & 120.782 & $-24,4$ \\
PR & 154.088 & 134.800 & 129.674 & 98.407 & 133.352 & 130.296 & 105.651 & $-31,4$ \\
PE & 96.076 & 48.440 & 29.430 & 24.825 & 21.277 & 44.553 & 53.022 & $-44,8$ \\
DF & 14.384 & 8.990 & 8.206 & 10.250 & 26.000 & 15.000 & 15.000 & 4,3 \\
RN & 7.972 & 6.840 & 4.422 & 4.068 & 1.748 & 5.203 & 14.100 & 76,9 \\
ES & 10.890 & 15.180 & 4.228 & 8.180 & 9.240 & 9.636 & 9.665 & $-11,2$ \\
PB & 2.270 & 2.626 & 2.256 & 1.308 & 1.070 & 3.560 & 4.084 & 79,9 \\
CE & 315 & 308 & 123 & 190 & - & - & 1.035 & 228,6 \\
PA & - & - & - & 525 & 700 & 700 & 700 & 33,3 \\
PI & 39 & 48 & 33 & 27 & 20 & 26 & 18 & $-53,8$ \\
\hline Total & 1.538 .929 & 1.646 .498 & 1.445 .989 & 1.654 .546 & 1.615 .311 & 1.540 .191 & 1.556 .885 & 1,2 \\
\hline
\end{tabular}

Fonte: SIDRA (2021).

Tabela 7: Evolução do rendimento médio da produção de cebola no Brasil. (Quilogramas por hectare).

\begin{tabular}{|c|c|c|c|c|c|c|c|c|c|}
\hline UF/ANO & 2013 & 2014 & 2015 & 2016 & 2017 & 2018 & 2019 & Média & Variação (\%) \\
\hline GO & 66.556 & 35.533 & 45.064 & 46.061 & 57.956 & 52.890 & 66.686 & 52.890 & 0,2 \\
\hline MG & 54.837 & 55.158 & 58.841 & 55.864 & 56.272 & 56.485 & 58.175 & 56.272 & 6,1 \\
\hline DF & 58.000 & 58.000 & 58.199 & 50.000 & 50.000 & 50.000 & 50.000 & 50.000 & $-13,8$ \\
\hline BA & 22.763 & 34.845 & 30.436 & 30.595 & 46.885 & 44.192 & 40.939 & 34.845 & 79,8 \\
\hline SP & 35.425 & 36.925 & 38.281 & 39.292 & 42.374 & 36.932 & 38.333 & 38.281 & 8,2 \\
\hline PA & - & - & - & 35.000 & 35.000 & 35.000 & 35.000 & 35.000 & 0,0 \\
\hline PE & 21.556 & 21.434 & 21.482 & 24.950 & 26.203 & 25.888 & 29.621 & 24.950 & 37,4 \\
\hline $\mathrm{ES}$ & 35.941 & 25.050 & 12.220 & 20.148 & 30.000 & 29.925 & 29.557 & 29.557 & $-17,8$ \\
\hline $\mathrm{SC}$ & 26.117 & 24.582 & 16.917 & 25.499 & 22.033 & 27.635 & 26.618 & 25.499 & 1,9 \\
\hline PR & 21.724 & 22.871 & 24.723 & 19.194 & 23.741 & 25.715 & 25.569 & 23.741 & 17,7 \\
\hline PB & 19.739 & 18.624 & 15.347 & 17.211 & 17.833 & 22.821 & 21.382 & 18.624 & 8,3 \\
\hline $\mathrm{CE}$ & 3.938 & 7.000 & 4.100 & 6.786 & - & - & 19.904 & 6.786 & 405,4 \\
\hline $\mathrm{RS}$ & 16.033 & 17.548 & 16.920 & 19.095 & 22.245 & 20.331 & 18.000 & 18.000 & 12,3 \\
\hline RN & 9.722 & 9.500 & 8.671 & 8.655 & 8.130 & 10.406 & 11.750 & 9.500 & 20,9 \\
\hline PI & 4.875 & 4.364 & 4.714 & 4.500 & 3.333 & 3.714 & 4.500 & 4.500 & $-7,7$ \\
\hline Total & 397.226 & 371.434 & 355.915 & 402.850 & 442.005 & 441.934 & 476.034 & & 19,8 \\
\hline
\end{tabular}


Atualmente no Registro Nacional de Sementes e Mudas (RENASEM), estão registradas aproximadamente 501 variedades de Allium cepa L., sendo 104 novas variedades entre os anos de 2015 e 2020 (MAPA, 2021).

A produção de sementes de cebola se dá predominantemente no estado do Rio Grande do Sul, que é responsável por 79,5\% da produção nacional (SIGEF, 2021). A região da fronteira sudoeste do RS é privilegiada para produção de sementes, por causa do fotoperíodo, temperatura e umidade, onde é alcançada uma produtividade média de $350 \mathrm{~kg}$ por hectare de sementes de cebola (Witter \& Blochtein, 2003).

No ano 2020/2021 o número total de campos inscritos por safra no Brasil girava em torno de 142, com uma área total de 281,22 ha, com uma produção bruta de 68,65 toneladas e produção estimada de 730,13 toneladas de sementes (SIGEF, 2021). A área plantada com cebola no Brasil em 2019 foi de 48.683 ha, com produção de 1.556 .885 t e rendimento médio de 25.499 kg.ha-1. A região Sul, com 683.684 toneladas, corresponde a 43,9\% dessa produção, seguida pelas regiões Sudeste e Nordeste com 373.417 e 310.982 toneladas, respectivamente. Nessas regiões, os maiores produtores são os estados de Santa Catarina (457.221 t), seguido da Bahia (242.807 t) e Minas Gerais (192.443 t) (SIDRA, 2021).

Apesar de sua importância econômica e social, o cultivo da cebola requer utilização de muitos insumos porque é uma planta exigente em solos férteis, necessitando de frequentes aplicações de fertilizantes. Além disso, a cebola é bastante suscetível à presença e proliferação de plantas daninhas, devido ao lento crescimento inicial, disposição ereta e forma cilíndrica de suas folhas, resultando em baixa capacidade de sombreamento (Soares et al., 2003).

A produção comercial de sementes de cebola é feita há várias décadas no Brasil, mas ainda assim o rendimento médio da produção é baixo, comparado a países com tradição na produção de sementes de cebola. Ainda é um desafio a produção de sementes de alta qualidade genética, fisiológica, física e sanitária no Brasil (Oliveira et al., 2004).

Segundo (Carvalho, 2020) trabalhos de monitoramento genéticos vêm sendo realizados pelo Instituto Agronômico de Pernambuco (IPA) e as cultivares utilizadas na região semiárida do Brasil vem proporcionando uma independência dos produtores em relação a materiais adaptados as condições ambientais bem como a geração de emprego. E ainda segundo (Carvalho, 2020) necessitam investimentos e desenvolvimento de tecnologias referentes ao manejo da fertirrigação, métodos alternativos de controle de pragas e doenças, agentes polinizadores, além do manejo orgânico na produção dessas sementes.

\subsection{Pimenta}

A pimenta-do-reino (Piper nigrum L) pertence à família Piperaceae, é uma planta perene nativa do Sul da Índia e foi introduzida no Brasil durante o século XVII, sendo difundida e intensificada no estado do Pará a partir de 1933 por imigrantes japoneses (Barbosa, 2002; EMBRAPA, 2011). A sua produção é distribuída no mundo inteiro, sendo a Etiópia campeã desse ranking, seguidos do Vietnã, Brasil e Indonésia. O Brasil aparece na $3^{\circ}$ posição (Tabela 8) e possui uma representatividade no valor das exportações de 6,33\% e importações de 0,14\% (FAOSTAT, 2021). No período de 2013 a 2019, a produção nacional aumentou $(158,6 \%)$ e a área colhida $(91 \%)$. (Tabelas 9 e 10$)$. 
Tabela 8: Ranking dos maiores produtores de pimenta (toneladas).

\begin{tabular}{ccccccccc}
\hline Ano/País & Etiópia & Vietnã & Brasil & Indonésia & Índia & Tajiquistão & Sri Lanka & Total \\
\hline 2013 & 288.994 & 125.023 & 42.312 & 91.000 & 53.000 & 216 & 26.730 & 627.275 \\
2014 & 204.410 & 151.761 & 42.339 & 87.400 & 51.000 & 868 & 27.847 & 565.625 \\
2015 & 322.655 & 176.789 & 51.739 & 81.501 & 65.000 & 280 & 31.226 & 729.190 \\
2016 & 425.395 & 216.432 & 54.430 & 86.334 & 55.000 & 1.075 & 32.145 & 870.811 \\
2017 & 347.284 & 252.576 & 79.106 & 87.991 & 72.000 & 3.046 & 35.142 & 877.145 \\
2018 & 328.841 & 262.658 & 101.624 & 88.949 & 66.000 & 33.847 & 48.253 & 930.172 \\
2019 & 374.413 & 264.854 & 109.401 & 88.949 & 66.000 & 47.107 & 41.429 & 992.153 \\
\hline Variação (\%) & 30 & 112 & 159 & -2 & 25 & 21.709 & 55 & 58 \\
\hline
\end{tabular}

Fonte: FAOSTAT (2021).

Tabela 9: Produção nacional em toneladas de pimenta-do-reino.

\begin{tabular}{ccccccccc}
\hline UF/Ano & 2013 & 2014 & 2015 & 2016 & 2017 & 2018 & 2019 & Variação (\%) \\
\hline ES & 6.728 & 7.597 & 13.863 & 12.801 & 34.591 & 60.555 & 62.633 & 830,9 \\
PA & 30.885 & 29.706 & 32.414 & 35.845 & 39.577 & 33.877 & 35.524 & 15,0 \\
BA & 4.049 & 4.467 & 4.696 & 4.916 & 3.792 & 5.870 & 9.691 & 139,3 \\
AL & 212 & 218 & 453 & 589 & 787 & 621 & 698 & 229,2 \\
MG & 6 & 18 & 37 & 53 & 104 & 333 & 516 & 8500,0 \\
RO & 41 & 46 & 42 & 88 & 89 & 178 & 177 & 331,7 \\
PB & 76 & 48 & 58 & 49 & 53 & 57 & 51 & $-32,9$ \\
RN & - & - & 13 & - & 30 & 55 & 45 & 246,2 \\
AM & 227 & 151 & 95 & 31 & 32 & 26 & 41 & $-81,9$ \\
MR & 63 & 54 & 44 & 41 & 38 & 38 & 20 & $-68,3$ \\
MT & 24 & 25 & 14 & 9 & 11 & 13 & 5 & $-79,2$ \\
\hline Total & 42.311 & 42.330 & 51.729 & 54.422 & 79.104 & 101.623 & 109.401 & 158,6 \\
\hline
\end{tabular}

Fonte: SIDRA (2021). 
Tabela 10: Área colhida de pimenta-do-reino no Brasil (Hectares).

\begin{tabular}{cccccccccc}
\hline UF/ANO & 2013 & 2014 & 2015 & 2016 & 2017 & 2018 & 2019 & Variação (\%) \\
\hline ES & 2.383 & 2.665 & 3.998 & 6.800 & 9.701 & 15.208 & 15.784 & 562 \\
PA & 13.948 & 14.236 & 15.891 & 16.668 & 16.139 & 15.683 & 15.745 & 13 \\
BA & 1.762 & 1.826 & 1.837 & 1.950 & 2.261 & 2.762 & 3.069 & 74 \\
AL & 69 & 72 & 134 & 181 & 298 & 273 & 271 & 293 \\
MG & 2 & 15 & 19 & 23 & 39 & 129 & 222 & 11.000 \\
RO & 39 & 45 & 42 & 64 & 56 & 93 & 94 & 141 \\
PB & 112 & 92 & 84 & 86 & 75 & 79 & 79 & -29 \\
MA & 53 & 48 & 42 & 37 & 33 & 30 & 19 & -64 \\
RN & - & - & 7 & - & 3 & 15 & 15 & 114 \\
AM & 62 & 38 & 26 & 8 & 10 & 9 & 14 & -77 \\
MT & 40 & 31 & 22 & 15 & 13 & 17 & 8 & -80 \\
\hline Total & 18.470 & 19.068 & 22.102 & 25.832 & 28.628 & 34.298 & 35.320 & 91 \\
\hline
\end{tabular}

Fonte: SIDRA (2021).

Atualmente no Registro Nacional de Sementes e Mudas (RENASEM), estão registradas aproximadamente 08 variedades de Pimentas, 02 novas variedades nos anos de 2015 a 2020. (MAPA, 2021). Em relação a campos de produção inscritos no SIGEF, nenhuma variedade de Piper nigrum foi encontrada, o que se pode inferir que as sementes dessa espécie são importadas. A área plantada com pimenta-do-reino no Brasil em 2019 foi de 35.998 ha, com produção de 109.401 t e rendimento médio de 2.324 kg.ha-1. A região Sudeste, com 63.149 toneladas, corresponde a 57,72\% dessa produção, seguida pelas regiões Norte e Nordeste com 35.742 e 10.505 toneladas, respectivamente. Nessas regiões, os maiores produtores são os estados de Espirito Santo (62.633 t), seguido do Pará (35.524 t) e Bahia (9.691 t) (SIDRA, 2021).

\section{Conclusão}

Apesar de uma demanda crescente de temperos no país, o Brasil possui poucos produtores explorando esse mercado, conforme pode ser observado a partir da quantidade de importação/exportação, bem como o número de campos de produção de sementes registrados no SIGEF. A produção de sementes está concentrada em poucas espécies e variedades. Ademais, a falta de estudos relacionados ao desenvolvimento de novas tecnologias e o domínio sobre as técnicas de cultivo de plantas condimentares tornam o Brasil ainda dependente de importações das sementes de espécies condimentares. Esse cenário ocorre principalmente em função da baixa representatividade das espécies condimentares no agronegócio, sendo grande parte das tecnologias e esforços direcionados às grandes culturas. Porém, a produção de sementes de condimentares pode ser uma alternativa potencial para geração de renda em pequenas propriedades, pois requer áreas menores para produzir um volume de sementes que atenda o mercado. Além disso, considerando que grande parte desses condimentos são utilizados diretamente na alimentação humana, há um apelo por um sistema de produção orgânicos e, para tal, é fundamental que as sementes também sejam produzidas nesse sistema. Há um longo caminho a ser percorrido, mas a partir da presente revisão, pode-se observar que existem espécies condimentares com potencial para exploração econômica da produção de sementes.

\section{Referências}

Andrade, J. L. R., Nunes, N. S. \& Gedanken, V. (2017) Plantas medicinais aromáticas e condimentares: produção e beneficiamento / Serviço Nacional de Aprendizagem Rural. Senar, 124p. 
Barbosa, F. B. C. (2002). Biotecnologia molecular e novo padrão de financiamento: Possibilidades para pesquisa da fusariose da pimenta-do-reino. Cadernos de Ciência e Tecnologia. 19(3), 429-49.

Brito, E. A. S. (2018). Consórcio de planta aromáticas com pimenta malagueta (Capsicum frutescens) como estratégia de manejo de pragas. Dissertação para obtenção do título Magister Scientiae UFV-MG. https://locus.ufv.br//handle/123456789/23207.

Carvalho, J. F., Pereira, R. F. \& Moreira, A. N. (2020). Produção de sementes de cebola em condições semiáridas. In Avanços Científicos e Tecnológicos nas Ciências Agrárias 6. Editora Atena, 35p.

Daflon, D. S. G., Freitas, M. S. M., Carvalho, A. J. C., Monnerat, P. H. \& Prins, C. L. (2014). Sintomas visuais de deficiência de macronutrientes e boro em coentro. Horticultura Brasileira. 32(1), 28-33. https://doi.org/10.1590/S0102-05362014000100005.

Factor, T. L., Purqueiro, L. F. V., Lima, S. L. \& Tivelli, S. W. (2008). Produção de salsa em função do período de cobertura com Agrotêxtil. In: Congresso Brasileiro de Olericultura. 26(2), 48p.

Faostat. (2021). http://www.fao.org/faostat/en/\#data/QC/visualize.

Fritsch, R. M. \& Friesen, N. (2002). Evolution, domestication, and taxonomy. In: Allium crop science: recent advances. Cabi, 5-30. https://doi.org/10.1079/9780851995106.0005.

Furlan, M. R. (1998). Ervas e temperos: cultivo e comercialização. Coleção Agroindústria. Sebrae/MT, 128p.

Goldman, I. L., Havey, M. J. \& Schroeck, G. (2000). History of public onion breeding programs and pedigree of public onion germplasm releases in the United States. Plant Breeding Reviews, 20(1), 67-103.

Gonçalves, L. F. C. (2016). Ensaio de competição entre cultivares de salsa em ambiente protegido. Trabalho de conclusão de curso em Agronomia, UnB-DF.

Haber, L. L. \& Clemente, F. M. V. T. (2013). Plantas aromáticas e condimentares: uso aplicado na horticultura. Embrapa, 168p.

Hata, F. T., Ventura, U. M., Paula, M. T., Shimizu, G. D., Paula, J. C. B., Kussaba, D. A. O. \& Souza, N. V. (2019). Intercropping garlic in strawberry fields improves land equivalent ratio and gross income. Ciência Rural 49 (12), 1-8p. https://doi.org/10.1590/0103-8478cr20190338.

Laws, B. (2013). 50 Plantas que mudaram o rumo da história. Tradução de Ivo Korytowski. 224p.

Leite, D. L. (2014). Produção de Sementes de Cebola. Embrapa Clima Temperado. Circular Técnica 142, 9p. http://www.infoteca.cnptia.embrapa.br/in foteca/handle/doc/992183.

Nepumoceno, R. (2005). O Brasil na rota das especiarias: o leva-e-traz de cheiros, as surpresas da nova terra. 176p.

Oliveira, A. P., Melo, P. C. T., Wanderley, L. J. G., Alves, A. U., Moura, M. F, N. \& Oliveira, A. N. P. (2007). Desempenho de genótipos de coentro em Areia. Horticultura Brasileira, 25(2), 252-255.

Oliveira, E. Q., Bezerra, N, F., Negreios, M. Z., Barros, Junior, A. P., Freitas, K. K. C., Silveira, L. M. \& Lima, J. S. S. (2005). Produção e valor agroeconômico no consórcio entre cultivares de coentro e de alface. Horticultura Brasileira. 23(2), 285-289.

Pereira, A. S., Shitsuka, D. M., Parreira, F. J., \& Shitsuka, R. (2018). Metodologia da pesquisa científica. UFSM. https://repositorio.ufsm.br/bitst ream/han dle/1/15824/Lic_Computacao_Metodologia-Pesquisa-Cientifica.pdf?sequence=1.

Pereira, R. C. A. \& Santos, O. G. (2013). Plantas Condimentares: Cultivo e Utilização. Fortaleza, CE: Embrapa Agroindústria Tropical, 55p.

Purquerio L. F. V. \& Melo, P. C. T. (2011). Hortaliças pequenas e saborosas. Horticultura Brasileira. 29(1), 1-1.

Rodrigues, A. P. D. C., Laura, V. A., Chermouth, K. S. \& Gadum, J. (2008). Absorção de água por semente de salsa, em duas temperaturas. Revista Brasileira de Sementes, 30(1), 49-54. https://doi.org/10.1590/S0101-31222008000100007.

Malhotra, S. K. \& Vashishtha, B. B. (2007). Production, Development, Quality and Export of Seed Spices, Nrcss, Ajmer, 373p.

Savage, J. (2017). Winter Spices in the Middle Ages https://ima.princeton.edu/2017/01/03/winter-spices-in-the-middle-ages/.

Shultes, R. E. \& Hofman, A. (2000). Plantas de los dioses: Orígenes del uso de los alucinógenos, 208p.

Sousa, T. V., Alkimim, E. R., David, A. M. S. S., Sá, J. R., Pereira, H. T. R. \& Mota, W. F. (2011). Época de colheita e qualidade fisiológica de sementes de coentro produzidas no norte de Minas Gerais. Revista Brasileira de Plantas Medicinais. Botucatu, 13(ed. especial), 591-597. https://doi.org/10.1590/S151605722011000500015

Tomchinsky, B. (2017). Prospecção de plantas aromáticas e condimentares no Brasil. Tese para obtenção do título de Doutor em Agronomia UNESP-SP. http://hdl.handle.net/11449/150786.

Torres, A. C., Dusi, A. N., Oliveira, R. \& Buso, J. A. (2001). Produção de alho-semente com alta qualidade fitossanitária mediante cultura de ápices caulinares. Embrapa Hortaliças. Circular Técnica 27, 8p.

Trupti, P. D., Gadhiya, A. D. \& Patel, G. D. (2018). A Review: Effect of Inter Cropping in Horticultural Crops. Int. J. Curr. Microbiol. App. Sci. 7(02), 15121520. https://doi.org/10.20546/ijcmas.2018.702.182.

Van eck, A. et al. (2020). Sauce it up: influence of condiment properties on oral processing behavior, bolusformationan ds enso rypercep tiono fs olidfoods. Food \& Function, 11(7), 6186-6201. 
Research, Society and Development, v. 10, n. 13, e95101321150, 2021

(CC BY 4.0) | ISSN 2525-3409 | DOI: http://dx.doi.org/10.33448/rsd-v10i13.21150

Veltri, A. L. A. (2017). Estudo de etnociências nas boas práticas agrícolas de plantas medicinais, aromáticas e condimentares do município de Botucatu. Dissertação para obtenção do título Mestre em Agronomia UNESP-SP. http://hdl.handle.net/11449/150062.

Witter, S. \& Blochtein, B. (2003). Efeito da polinização por abelhas e outros insetos na produção de sementes de cebola. Pesquisa Agropecuária Brasileira. 38(12), 1395-1407. 